\title{
Estudo comparativo entre agentes reticulantes para possível aplicação no tratamento do ceratocone
}

\section{Comparative study between reticulating agents for possible application in the treatment of keratoconus}

Camila Melo Gadelha Pereira Diniz ${ }^{1}$, Josué da Silva Buriti ${ }^{1}$, Rossemberg Cardoso Barbosa ${ }^{1}$, Marcus Vinícius Lia Fook ${ }^{1}$

\footnotetext{
${ }^{1}$ Unidade Acadêmica de Engenharia de Materiais, Universidade Federal de Campina Grande, Rua Aprígio Veloso, 882, Bodocongó, CEP: 58429-140, Campina Grande, Paraíba, Brazil.

e-mail: camilamgpd@gmail.com, josueburiti@gmail.com,rcbvet@gmail.com, viniciusliafook@yahoo.com.br
}

\begin{abstract}
RESUMO
Com o presente estudo, objetivou-se comparar a técnica padrão do CXL com o tratamento utilizando a genipina como reticulante, com e sem desepitelização da córnea. Comparou-se propriedades químicas, mecânicas e morfológicas de córneas de suínos submetidas aos tratamentos de crosslinking com riboflavina e genipina, bem como comparar a citotoxicidade das soluções reticulantes, para possível aplicação no ceratocone. Para isso, as córneas foram divididas em quatro grupos: riboflavina e UVA conforme tratamento padrão, genipina com e sem desepitelização e controle sem reticulante. As córneas foram submetidas às caracterizações de MO, MEV, ensaio de tração, dilatometria e intumescimento. A citotoxicidade foi realizada comparando o padrão com a genipina. Os resultados demonstram maior resistência à tração e maior grau de intumescimento quando a genipina foi aplicada após desepitelização da córnea. As inclinações descendentes na curva de dilatometria mostram-se lineares no UV-CXL e controle, e com a genipina, a contração tende a ficar mais acentuada em temperaturas maiores. Pela MEV foi verificada uma linha de demarcação no estroma corneano tratado com UV-CXL ou genipina e a MO exibiu padrões diversos de luz transmitida nos diferentes grupos, demonstrando maior opacidade no UV-CXL. O crosslinking do colágeno corneano induzido pela genipina $0,1 \%$ produz aumento na resistência da córnea sem provocar opacidade corneana considerável, podendo ser útil no tratamento do ceratocone. O potencial de citotoxicidade aos fibroblastos se mostrou semelhante entre os métodos de reticulação.
\end{abstract}

Palavras-chave: crosslinking, córnea, genipina, riboflavina, ceratocone.

\section{ABSTRACT}

With the present study, we aimed to compare the standard technique of CXL with the treatment using genipine as a crosslinker, with and without corneal de-epithelialization. The chemical, mechanical and morphological properties of pig corneas submitted to crosslinking treatments with riboflavin and genipine were compared, as well as to compare the cytotoxicity of the crosslinking solutions for possible application in keratoconus. The corneas were divided into four groups: riboflavin and UVA according to standard treatment, genipina with epithelium off, genipina with epithelium on and control without crosslinker. In this way, the corneas were submitted to the characterizations and analyzed by the following techniques: OM, SEM, traction test, dilatometry and swelling. The cytotoxicity analysis was performed comparing the standard treatment with genipine. The results demonstrate a greater tensile strength, as well as more swelling when genipine was applied to the epithelium off corneal surface. The downward slopes in the dilatometry curve are linear in the UV-CXL and control, and with genipine, the contraction tends to become more pronounced at higher temperatures. By MEV was able to demonstrate a demarcation line in the corneal stroma treated with UV-CXL or genipine and OM exhibits different patterns of transmitted light in the different groups, demonstrating greater UV-CXL opacity. The corneal collagen crosslinking induced by $0.1 \%$ genipine produces an 
increase in corneal resistance without causing considerable corneal opacity and may be useful in the treatment of keratoconus. The potential for cytotoxicity to fibroblasts was similar between crosslinking methods.

Keywords: crosslinking, cornea, genipin, riboflavin, keratoconus.

\section{INTRODUÇÃO}

O ceratocone é uma desordem da córnea caracterizada por ectasia e consequente aumento de sua curvatura central, geralmente bilateral e progressiva [1]. Observações quanto a menor progressão do ceratocone em pacientes com diabetes e maior idade levou à idéia de que o crosslinking (CXL) natural das fibras de colágeno da córnea poderia resultar em fortalecimento e enrijecimento dos tecidos [2]. Isso levou ao desenvolvimento da reticulação do colágeno corneano, um processo, no qual, uma combinação de um fotoindutor, luz ultravioleta (UV) e uma reação fotoquímica leva a indução de radicais livres, que conduz a uma ligação química entre as fibras de colágeno.

A reticulação polimérica é um processo que ocorre quando cadeias poliméricas lineares ou ramificadas são interligadas por pontes de ligações, formando uma rede polimérica tridimensional, processo também conhecido como crosslinking (formação de ligações cruzadas) [3, 4]. O crosslinking pode ser induzido através de diferentes métodos, como glicação não enzimática, irradiação utilizando luz ultravioleta com ou sem a associação de fotossensibilizantes e por reações de aldeído [5].

Seiler et al. [6] demonstrou que o uso de irradiação ultravioleta A (UVA) associada à riboflavina (vitamina B2) para indução de crosslinking de colágeno da córnea (CXL) apresentar-se-ia como uma técnica promissora; que, de fato, vem sendo divulgada através das experiências clínicas de pesquisadores do mundo inteiro. O principal objetivo do uso do crosslinking é conter a progressão do ceratocone. Logo, o melhor candidato ao tratamento é o indivíduo com sinais claros de progressão da doença. Até o momento, não existem critérios definitivos para a progressão do ceratocone, porém os parâmetros a serem considerados são a mudança do erro refrativo, piora da acuidade visual, bem como progressão nos valores encontrados nas topografias e tomografias da córnea [7].

A riboflavina atua como fotoindutor e, submetida à exposição à radiação UVA, gera radicais livres que promovem ligações cruzadas no colágeno corneano, além de promover proteção à radiação às estruturas intraoculares como cristalino e retina. No entanto, a riboflavina é uma molécula grande e necessita da desepitelização para alcançar o colágeno do estroma corneano. Muitas técnicas na promoção de crosslinking (CXL) do colágeno corneano vêm sendo estudadas no intuito de se evitar a desepitelização corneana, mas nenhuma demonstra a mesma eficácia da técnica padrão.

Outro agente reticulante que vem sendo muito utilizado é a genipina, alvo de várias pesquisas na reticulação de biomateriais [8-11]. Há poucos estudos considerando a genipina como reticulante do colágeno corneano $[12,13]$. Devido às suas propriedades físicas, químicas e biológicas, se mostra promissora nesse intuito, e teria a vantagem de não utilizar radiação ultravioleta, agente físico necessário no protocolo padrão do CXL.

Assim, o objetivo deste trabalho foi investigar e comparar a influência de agentes reticulantes como a riboflavina + UVA e solução de genipina $0,1 \%(\mathrm{~m} / \mathrm{v})$, em propriedades químicas, mecânicas e morfológicas de córneas de porcos, bem como comparar a citotoxicidade das soluções reticulantes, para possível aplicação no tratamento do ceratocone.

\section{MATERIAIS E MÉTODOS}

Trinta e dois olhos de suínos, com córneas claras, isentas de qualquer defeito ou opacidade, foram coletados logo após o abate, transportados em caixa térmica, em temperatura de 2 a $8{ }^{\circ} \mathrm{C}$ e usados, dentro de 8 a $10 \mathrm{~h}$ post-mortem, mantidos sob refrigeração para separação das córneas. Vale salientar que as córneas foram transportadas em caixas térmicas com gelo.

Posteriormente, as córneas foram separadas em quatro grupos, denominados grupo 1 (C1 - córneas submetidas a aplicação de riboflavina + UVA de acordo com o tratamento de crosslinking em humanos, segundo protocolo de Dresden - Figura 1), grupo 2 (C2 - córneas submetidas a aplicação da solução de genipina $0,1 \%$ por $30 \mathrm{~min}$, sendo aplicada uma gota da solução a cada $5 \mathrm{~min}$, após a desepitelização da córnea Figura 2a), grupo 3 (C3 -córneas, com epitélio íntegro, sendo aplicada uma gota da solução de genipina $0,1 \%$ a cada 5 min - Figura 2b) e grupo 4 (C4 - córneas do grupo controle, com epitélio íntegro, sem reticulante Figura 2c).

Após cada tratamento, botões córneo-esclerais foram retirados dos globos oculares. Em seguida, foram preparadas faixas verticais centrais para os ensaios de tração e dilatometria e, foram trepanados botões 
corneanos (7,75 mm centrais) para os ensaios de grau de intumescimento, microscopia óptica e microscopia eletrônica de varredura. Os botões corneanos foram secos com papel toalha e submetidas às caracterizações (Figura 3).
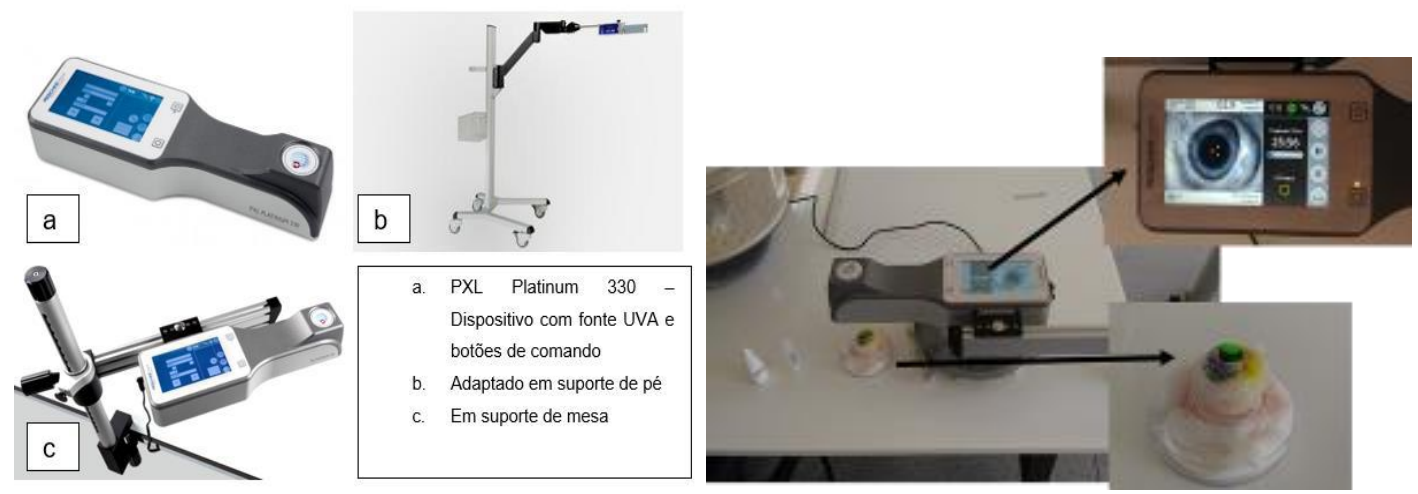

Figura 1: Equipamento utilizado como fonte UVA para o CXL durante a fase experimental [14].
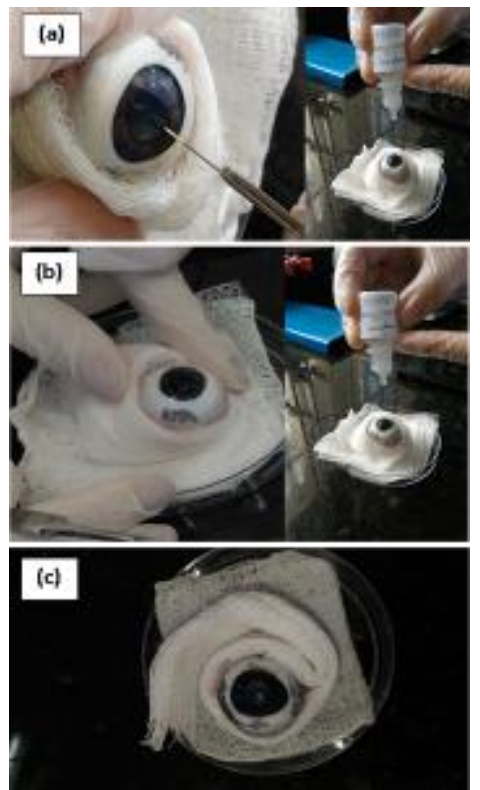

Figura 2: Aplicação da genipina e Grupo controle [(a) grupo C2 (genipina 0,1\% sem epitélio); (b) grupo C3 (genipina $0,1 \%$ com epitélio)]; (c) grupo C4 (controle, sem reticulante).

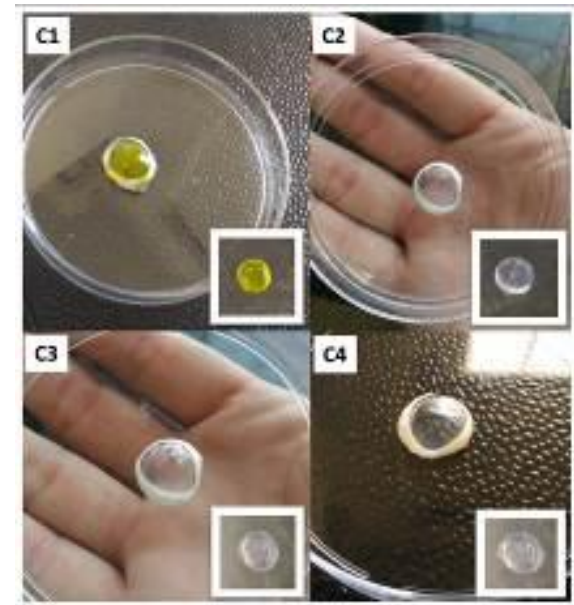

Figura 3: Botões Córneo-esclerais e botões corneanos: C1 (Riboflavina + UVA); C2 (genipina 0,1\% sem epitélio); C3 (genipina $0,1 \%$ com epitélio); C4 (controle, sem reticulante). 
Todo o experimento foi baseado nos critérios preconizados pela Association for Research in Vision and Ophthalmology (ARVO [15]) para o uso de animais em pesquisa oftálmica e na Resolução do Conselho Nacional de Controle de Experimentação Animal - Resolução Normativa N 30 (CONCEA [16]) diretriz VI 6.1.10 na letra $b$, que orienta sobre uso de cadáveres, parte deles ou amostras biológicas.

Os ensaios de tração foram realizados numa máquina de ensaio universal, da marca Instron, modelo 3366, com célula de carga de $10 \mathrm{kN}$, velocidade de $1,0 \mathrm{~mm} / \mathrm{min}$, com 3 amostras de aproximadamente 15 $\mathrm{mm}$ de comprimento e $4 \mathrm{~mm}$ de largura. A distância entre as garras foi de $5 \mathrm{~mm}$. O ensaio teve como objetivo determinar o módulo de elasticidade $(\mathrm{MPa})$, deformação máxima (\%) e a tensão (MPa) das córneas. A dilatometria foi utilizada para medir a variação de comprimento das córneas quando submetida a um programa controlado de temperatura. Foi utilizado um dilatômetro da Netzsch modelo 402 C. A faixa de temperatura utilizada foi de 20 a $40{ }^{\circ} \mathrm{C}$ com razão de aquecimento de $2{ }^{\circ} \mathrm{C} / \mathrm{min}$.

A microscopia óptica das córneas foi realizada em um microscópio óptico Hirox de reflexão e transmissão com acessórios 2D e variação de 50X-400X, acoplado a uma estação de análise de imagens, com aumento de 300x, da parte longitudinal. Já para obter as micrografias por microscopia eletrônica de varredura, foi utilizado um equipamento de bancada, modelo TM-1000, da marca Hitachi, com aumento de até 10000x, com profundidade de foco de $1 \mathrm{~mm}$, resolução de $30 \mathrm{~nm}, 15 \mathrm{KV}$, baixo vácuo e pressão variada de 1 a 270 $\mathrm{Pa}$, sem recobrimento metálico. As imagens foram obtidas com aumento de 400x.

Para avaliar o grau de intumescimento das córneas dos quatro grupos, as mesmas foram inicialmente pesadas em uma balança analítica e mantidas em solução de tampão fosfato salino (PBS) por 24 h. Em seguida, foram retiradas da solução, colocadas sobre papel filtro para retirar o excesso de solução e pesadas novamente. O grau de intumescimento de cada amostra no tempo 't', foi calculado de acordo com a Equação 1. Todas as amostras passaram por medições antes e após o intumescimento para verificar o quanto o sistema variou, onde 'Wt' é a massa da amostra no tempo 't' e 'Wo' é a massa inicial da amostra.

$$
G I=\frac{W t-W o}{W o} x 100
$$

A avaliação da citotoxicidade in vitro foi realizada pelo teste de avaliação da viabilidade celular dos fibroblastos L929 por MTT [brometo de 3-(4,5dimetiltiazol-2-il)-2,5-difenil-tetrazólio] de acordo com a ISO 10993-5:2009 (ISO [17]). Os parâmetros de avaliação observados são a porcentagem de morte celular e a IC50 (concentração do produto que inibe 50\% do crescimento celular). Utilizou-se igual volume das soluções de genipina $0,1 \%$ e riboflavina, sendo que as amostras que foram submetidas à riboflavina também foram expostas à radiação UVA durante 30 min. Ambas as amostras estiveram nas mesmas condições ambientais, durante os 30 min de experimento, em câmara de fluxo laminar.

\section{RESULTADOS E DISCUSSÕES}

\subsection{Ensaios de tração}

Os resultados dos ensaios de tração das córneas, para três corpos de prova de cada grupo, estão apresentados na Figura 4 e Tabela 1.

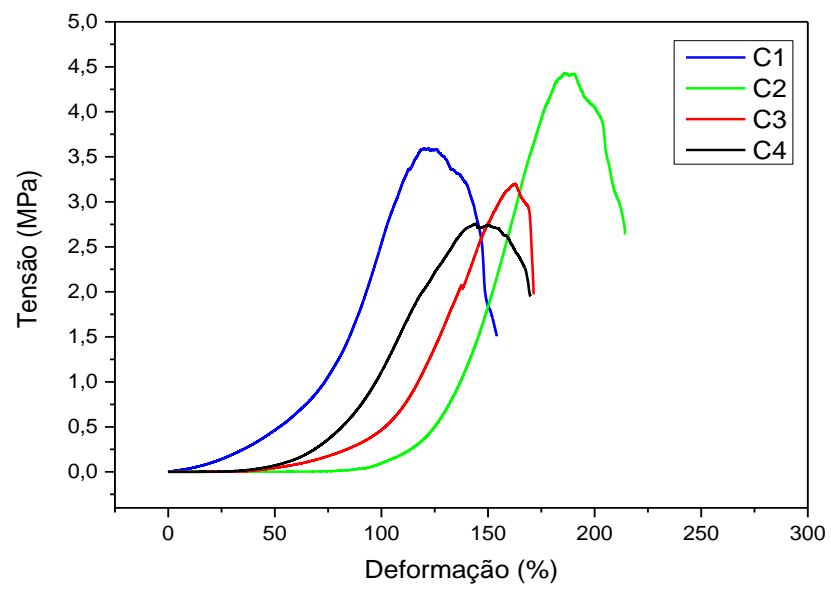

Figura 4: Curvas tensão x deformação das córneas dos grupos C1, C2, C3 e C4. 
Tabela 1: Dados das propriedades mecânicas com base nas curvas tensão x deformação das córneas dos grupos C1, C2, $\mathrm{C} 3$ e C4.

\begin{tabular}{c|c|c|c}
\hline Amostra & Módulo de elasticidade (Mpa) & Deformação máxima (\%) & Tensão (Mpa) \\
\hline $\mathrm{C} 1$ & $0,039 \pm 0,0042$ & $116,00 \pm 7,61$ & $3,29 \pm 0,749$ \\
\hline $\mathrm{C} 2$ & $0,038 \pm 0,0014$ & $186,89 \pm 5,64$ & $4,50 \pm 0,007$ \\
\hline $\mathrm{C} 3$ & $0,035 \pm 0,0099$ & $162,48 \pm 2,62$ & $3,20 \pm 0,140$ \\
\hline $\mathrm{C} 4$ & $0,033 \pm 0,0035$ & $162,86 \pm 18,51$ & $2,90 \pm 0,250$ \\
\hline
\end{tabular}

Verificou-se que os grupo $\mathrm{C} 1$ e C2 apresentaram maior resistência à tração $(3,29 \pm 0,749 \mathrm{MPa} ; 4,50 \pm$ 0,007 MPa respectivamente) quando comparados ao grupo C3 (3,20 $\pm 0,140 \mathrm{MPa})$. Este fato pode estar relacionado ao tecido epitelial, presente nas córneas dos grupos $\mathrm{C} 3$ e $\mathrm{C} 4$, podendo representar uma barreira à maior penetração do reticulante no estroma corneano, diminuindo, assim, a formação de ligações cruzadas.

A deformação máxima das córneas do grupo 1 se apresentou menor do que a dos demais grupos $(116,0 \pm 7,61 \%)$. Córneas submetidas ao tratamento com genipina $0,1 \%$, após desepitelização, apresentam maior plasticidade, com deformação máxima de $186,89 \pm 5,64 \%$. A necessidade de aumentar a tensão para dar continuidade à deformação plástica do material decorre de um fenômeno denominado encruamento. A partir da região de escoamento, o material entra no campo de deformações permanentes, onde ocorre endurecimento por deformação a frio. Esse fenômeno ocorre em função da interação entre discordâncias e das suas interações com outros obstáculos, como solutos, contornos de grãos, entre outros, que impedem a livre movimentação das discordâncias.

À medida que os planos cristalinos escorregam e deslizam entre si, permitindo o escoamento, eles são continuamente travados devido à ancoragem desses planos pelas discordâncias que atingem os contornos de grão. Conforme mais e mais planos cristalinos são travados, é preciso uma energia cada vez maior para que os planos que ainda possuem liberdade de movimentação continuem a deslizar, e, consequentemente, dando continuidade à deformação plástica, até o limite em que a fratura tem início [18].

As propriedades biomecânicas da córnea se encontram intimamente relacionadas à sua estrutura, com especial envolvimento de seu estroma [19]. Sua elasticidade depende basicamente do arranjo estabelecido pelas fibras colágenas, e varia conforme a direção e região em que a variável é mensurada [20]. Estudos têm demonstrado que a córnea humana apresenta comportamento elástico não-linear [19]. Além disso, o módulo de elasticidade da córnea varia direccionalmente e regionalmente. Elevados módulos de elasticidade são observados meridionalmente, em regiões centrais e paracentrais da córnea, e circunferencialmente, próximo ao limbo [21].

Vale salientar que a espessura média das córneas, neste estudo, para os grupos $\mathrm{C} 1, \mathrm{C} 2, \mathrm{C} 3$ e C4 foram de $880 \pm 43 \mu \mathrm{m}, 1000 \pm 99 \mu \mathrm{m}, 1160 \pm 197 \mu \mathrm{m}$ e $1210 \pm 64 \mu \mathrm{m}$. Realizaram-se as medidas nas córneas que foram submetidas ao ensaio de tração.

Em estudo prévio, com objetivo de avaliar o efeito da genipina, como reticulante natural em córneas porcinas, em diversas concentrações, os autores puderam perceber aumento do módulo de elasticidade e rigidez da córnea, de um modo dose-dependente [13]. Os valores de módulo de elasticidade foram bastante superiores ao encontrado no presente estudo, o que pode ser explicado pela penetração da genipina pelas laterais das faixas corneanas confecionadas antes da aplicação do reticulante. No presente estudo, o método de aplicação do tratamento, antes da confecção de botões corneanos ou faixas córneo-esclerais, simula ou assemelha-se, mais perfeitamente ao que ocorre no tratamento in vivo.

Ao se comparar a rigidez corneana, em condições fisiológicas, em olhos tratados com genipina 0,25\% ou tratados com associação de riboflavina + UVA, o efeito do crosslinking foi similar [12]. No presente estudo, verificamos maior limite de resistência à tração das córneas tratadas com genipina após despitelização, no entanto, essas mesmas córneas apresentam uma deformação máxima mais elevada do que as submetidas ao protocolo padrão de crosslinking corneano.

Pode-se entender que, ao utilizar concentração menor da genipina, permitimos ainda a presença de regiões sem crosslinking do colágeno, aumentando a deformação máxima das córneas tratadas com essa concentração. Estudos comparando diversas concentrações da genipina com o tratamento com riboflavina e UVA podem ser realizados para tornar essa hipótese mais firme. 


\subsection{Dilatometria}

A Figura 5 apresenta as curvas dilatométricas das córneas dos grupos C1, C2, C3 e C4, obtidas sob razão de aquecimento de $2^{\circ} \mathrm{C} / \mathrm{min}$.
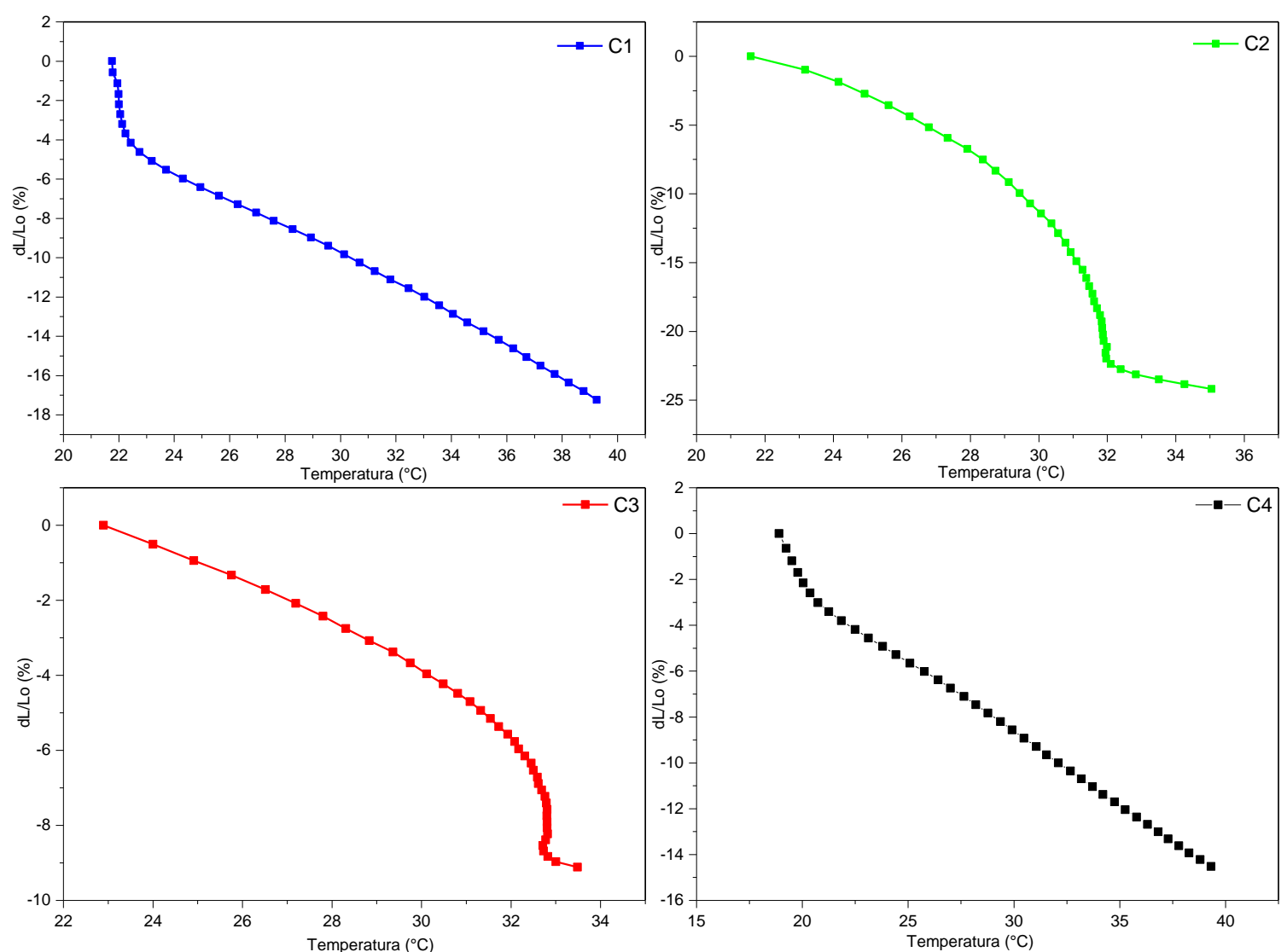

Figura 5: Curvas dilatométricas das córneas dos grupos $\mathrm{C} 1, \mathrm{C} 2, \mathrm{C} 3$ e $\mathrm{C} 4$, obtidas sob razão de aquecimento de $2^{\circ} \mathrm{C} / \mathrm{min}$.

Em relação aos resultados dos ensaios de dilatometria, o maior percentual de contração foi das córneas do grupo C2 (genipina 0,1\% sem epitélio), seguidas das córneas do grupo C1, corroborando os resultados do ensaio de tração, demonstrando maior resistência mecânica nos grupos C1 e C2 (córneas desepitelizadas previamente a aplicação da riboflavina e UVA (C1) ou genipina $0.1 \%$ (C2), onde, provavelmente, o efeito do crosslinking foi maior.

Deve-se considerar também que essas diferenças na amplitude de contração das córneas, bem como das diferentes temperaturas envolvidas nas contrações, entre os grupos, podem ser um sinal dos diferentes níveis ou tipos de ligações cruzadas, ou ainda das diferentes formas de enovelamento da tripla-hélice do colágeno, o que pode ser atribuído à distribuição heterogênea das ligações cruzadas, causada pela difusão lenta dos reagentes no tecido [22]. Não foi encontrado nas bases de dados e pesquisa utilizados, estudos de dilatometria em córneas, sejam elas de qualquer espécie.

\subsection{Microscopia óptica}

A Figura 6 apresenta as micrografias obtidas por MO das córneas (região central), com representações longitudinais de 300x de aumento, com o objetivo de investigar o comportamento do material, quanto a interação com a luz. 


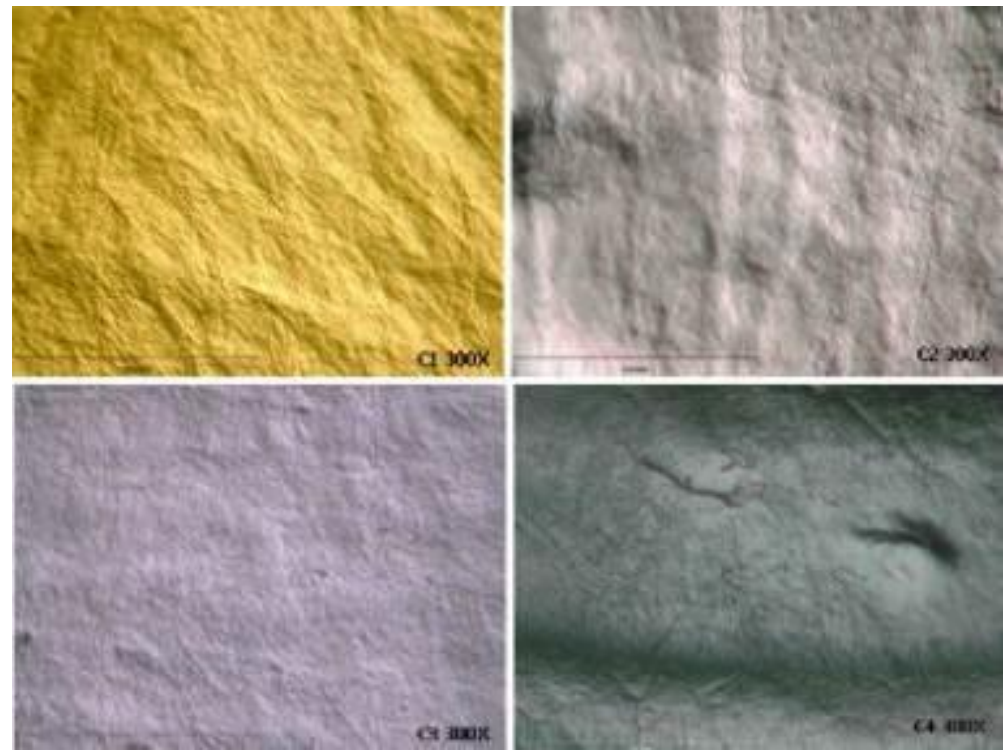

Figura 6: Micrografias obtidas por MO das córneas (região central) dos grupos $\mathrm{C} 1, \mathrm{C} 2, \mathrm{C} 3$ e $\mathrm{C} 4$, com representações longitudinais de 300x de aumento.

Pela análise das micrografias, percebe-se uma coloração amarelada para a córnea submetida ao tratamento com riboflavina + UVA (C1), coloração discretamente azulada para as córneas submetidas ao tratamento com genipina $0,1 \%(\mathrm{C} 2$ e $\mathrm{C} 3)$ e mais transparentes ou acinzentadas para a córnea do grupo controle (C4).

Verifica-se que houve um aumento da rugosidade e da densidade nas amostras dos grupos C1 e C2, onde provavelmente houve maior reticulação, quando comparada com a superfície da C3 e C4. Este fato corrobora com o observado nos ensaios mecânicos, as quais $(\mathrm{C} 1$ e $\mathrm{C} 2)$ apresentaram maior resistência à tração.

Curiosamente, verificou-se por fotografias, a comparação da transparência e visualização de imagens de letras através dos botões corneanos dos diversos grupos, comparados ao controle (Figura 7). As córneas submetidas à genipina demonstraram melhor visualização das letras quando comparadas às submetidas ao protocolo padrão. Análises de transmitância podem ser realizadas para melhor investigação da transparência dessas córneas.
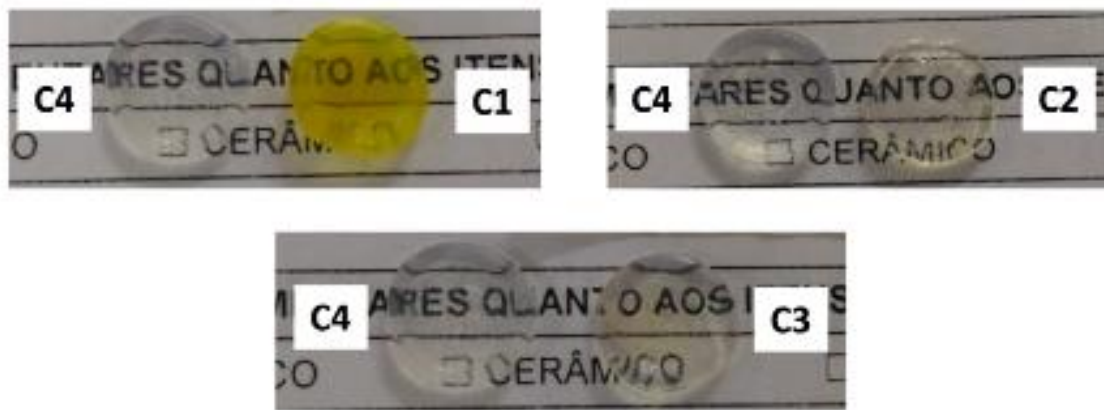

Figura 7: Fotografias dos botões corneanos (C1, C2, C3), comparando-os, em transparência, com o controle (C4).

Através da caracterização realizada por microscopia óptica, pode-se perceber as diferentes colorações das amostras. A técnica utilizada foi a de transmissão. A cor dos materiais observados em transmissão depende claramente da absorção seletiva. Um material iluminado por luz branca e que absorve seletivamente determinada cor, será visto com sua cor complementar. Para o caso de materiais opacos, o processo é mais complicado. A grande maioria dos materiais coloridos apresenta coloração pigmentada, na qual a luz penetra uma certa distância durante a qual sofre absorção seletiva, e por um mecanismo (que pode ser reflexão, refração, difração ou espalhamento) emerge da sua superfície a sua cor complementar [23].

No tratamento padrão de crosslinking do colágeno corneano, o estroma da córnea deve ficar embebido com a solução de riboflavina, tanto para que haja o efeito fotoquímico desejado quanto para que a mesma exerça função de proteção de estruturas intra-oculares contra os danos da radiação ultravioleta. Isso, por si só, 
já é capaz de provocar a coloração amarelada da córnea. Sabe-se que o crosslinking "padrão" pode estar associado ao aparecimento de opacidade corneana ou "haze" em 57\% dos pacientes submetidos ao mesmo [24] e que esse haze é maior no primeiro mês, apresenta um platô em 3 meses, e reduz significativamente entre 3 e 12 meses do período pós-operatório [25]; estende-se até $60 \%$ da profundidade do estroma e, apesar da sua natureza não ser totalmente conhecida, pode estar associado a uma transformação anômala dos fibroblastos e morte dos ceratócitos e a profundidade do efeito do crosslinking [26, 27].

Esse haze, ou opacidade da córnea, é tido como complicação do UV-CXL, visto que pode provocar, embora transitóriamente, uma indesejada diminuição da acuidade visual. O tratamento com genipina pode ser uma melhor alternativa ao tratamento no que diz respeito ao grau de transparência corneana pós-tratamento. Estudos, com essa finalidade, podem trazer informações adicionais.

\subsection{Microscopia Eletrônica de Varredura (MEV)}

A Figura 8 apresenta as micrografias obtidas por MEV das córneas dos grupos C1, C2, C3 e C4, com representações transversais (corte central) e 400x de aumento.
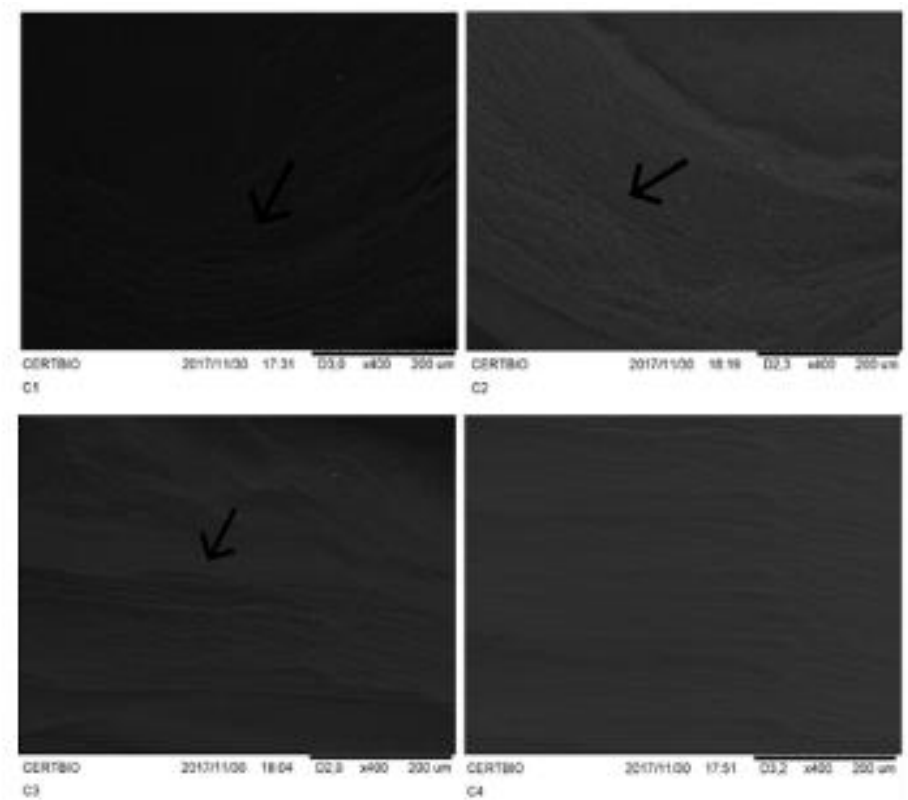

Figura 8: Micrografias obtidas por MEV das córneas dos grupos $\mathrm{C} 1, \mathrm{C} 2, \mathrm{C} 3$ e $\mathrm{C} 4$, com representações transversais (corte central) e 400x de aumento.

As micrografias obtidas por MEV, demonstraram uma linha de demarcação no estroma corneano. A nova estruturação das fibras colágenas proporciona um novo arranjo molecular, com aproximação das fibrilas adjacentes como visualizado por microscopia de fluorescência confocal, no trabalho de BOTTÓS et al. [28].

Após o tratamento convencional com riboflavina + UVA, tem sido detectada uma linha de demarcação no estroma corneano, ao exame de biomicroscopia em lâmpada de fenda, já em 2 semanas do período pós-operatório a uma profundidade de aproximadamente $300 \mu \mathrm{m}$ no estroma corneano [29]. Essa linha de demarcação também pode ser detectada utilizando-se microscopia confocal e tomografia de coerência óptica do segmento anterior ocular, representando a efetividade do crosslinking do colágeno corneano [30]. Assim, pode-se inferir que a genipina $0,1 \%$ administrada na córnea com ou sem epitélio, foi capaz de provocar alterações no estroma corneano, compatíveis com o crosslinking do colágeno corneano, semelhante ao produzido pelo tratamento convencional com riboflavina + UVA. 


\subsection{Grau de intumescimento}

A Figura 9 apresenta os resultados de grau de intumescimento (\%) das córneas dos grupos C1, C2, C3 e C4.

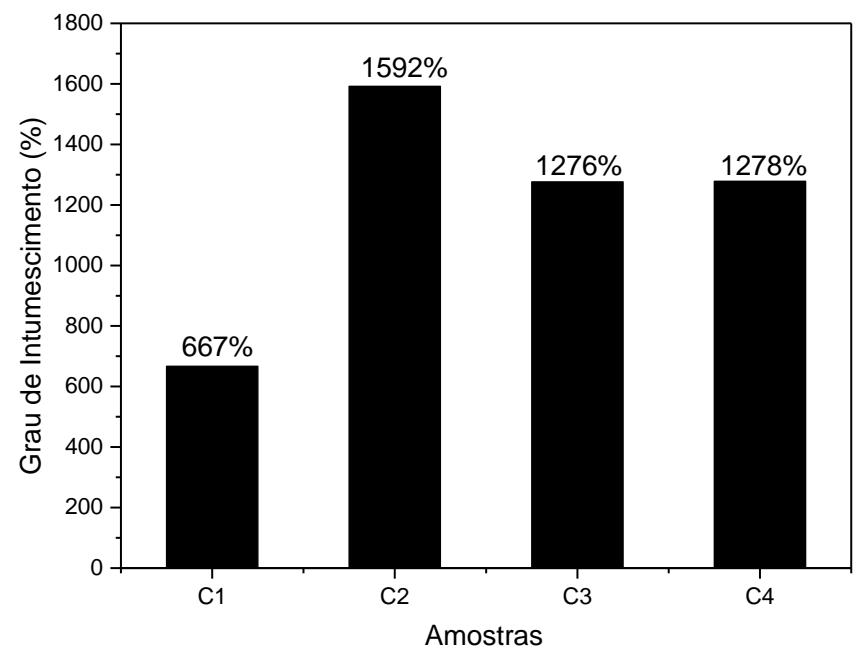

Figura 9: Micrografias obtidas por MEV das córneas dos grupos C1, C2, C3 e C4, com representações transversais (corte central) e 400x de aumento.

Analisadas em relação ao grau de intumescimento, o tratamento padrão com riboflavina e UVA levaram a um menor grau de intumescimento, o que denota uma maior força de interação ou compactação entre as camadas de fibras de colágeno, no estroma corneano, impedindo a entrada da solução de PBS no mesmo.

Sabe-se que córneas humanas, portadoras de ceratocone, mostraram uma razão alterada dos proteoglicanos dermatan sulfato/keratan sulfato [31] e pouco se entende sobre propriedades de intumescimento da córnea nos portadores de ceratocone. Vimos, em outra oportunidade, que o crosslinking do colágeno corneano, com riboflavina + UVA, provoca uma diminuição na síntese dos glicosaminoglicanos [32]. Os glicosaminoglicanos possuem alta quantidade de carga negativa e por isso acabam atraindo uma nuvem de cátions, onde o mais atraído é o sódio que traz com ele moléculas de água. Essa capacidade dos glicosaminoglicanos de atrair cátions e água, confere aos proteoglicanos a função de dar a matriz extracelular uma característica hidratada. É possível que a genipina exerça menor influência na quantidade de glicosaminoglicanos presentes na matriz extracelular do estroma corneano.

A reticulação do colágeno da córnea (CXL) com riboflavina $0,1 \%$ e ultravioleta-A (UVA) pode fortalecer as ligações interfibrilares do estroma, reduzindo o espaço potencial para o acúmulo de fluido nas córneas. Avaliação objetiva da hidratação da córnea, após CXL do colágeno corneano, com riboflavina e UVA, demonstrou, na microscopia óptica, que não houve hidratação nos $351 \mu \mathrm{m}$ anteriores do estroma da córnea, região onde ocorreu o efeito biomecânico do crosslinking [33].

Interessante estudo do efeito do CXL com riboflavina + UVA na estrutura e no comportamento hidrodinâmico [34] demonstrou que há redução significante da espessura após aplicação de riboflavina - irradiada ou não; mas que, após lavagens e saída do dextran, iniciou-se o estudo e a hidratação máxima alcançada não diferiu entre tratadas e não tratadas e, como não houve mudanças ultraestruturais no estroma corneano (espaçamento interfibrilar, diâmetro da fibra e espaçamento entre as moléculas de colágeno) significantes, sugeriuse que é provável que as ligações cruzadas formadas durante a terapia com riboflavina/UVA ocorram predominantemente na superfície da fibrila de colágeno com a rede de proteínas que circundam o colágeno (proteoglicanos). A diferença observada, no grau de intumescimento, no presente estudo, nos traz a curiosidade de onde se formam essas ligações quando o agente reticulante utilizado é a genipina.

\subsection{Citotoxicidade}

A Figura 10 apresenta os resultados de citotoxicidade nas amostras de fibroblastos L929 submetidas à exposição à genipina $0,1 \%$ ou a associação de riboflavina + UVA. 


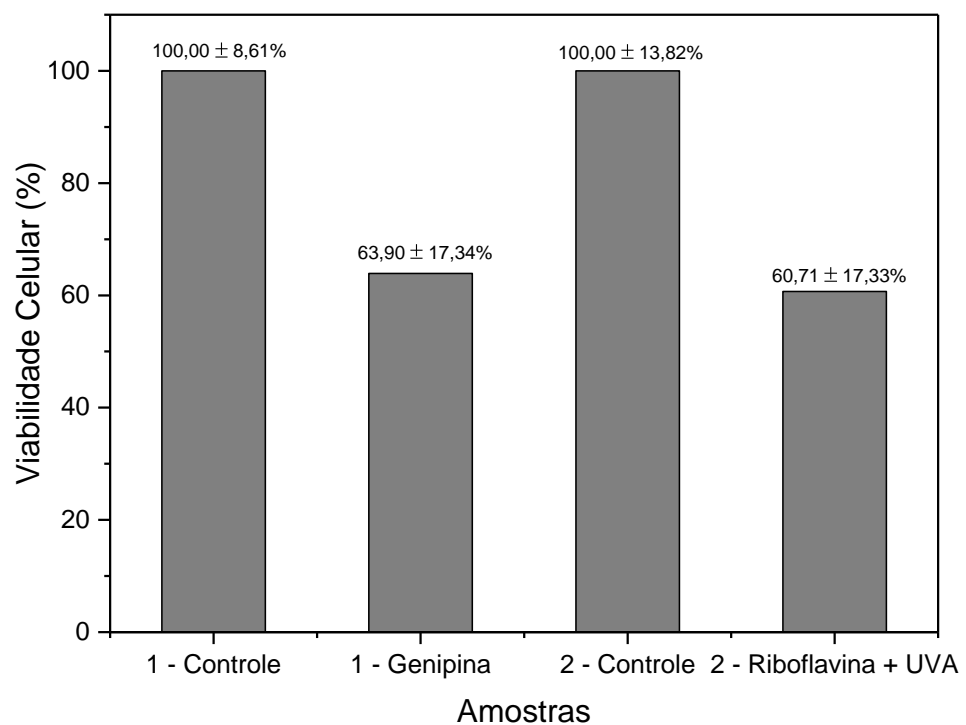

Figura 10: Análise de citotoxicidade de genipina $0,1 \%$ ou associação de riboflavina + UVA aos fibroblastos L929.

Com base na avaliação da viabilidade celular de fibroblastos L929, em cultura, observou-se que ambos os tratamentos avaliados são potencialmente citotóxicos. Utilizando-se a irradiação padrão, da superfície corneana, com a fluência de $3 \mathrm{~mW} / \mathrm{cm}^{2}$, o efeito citotóxico atinge a profundidade de $300 \mu \mathrm{m}$ em córneas humanas [35].

Sabe-se que o estroma é despovoado de ceratócitos até essa profundidade de $300 \mu \mathrm{m}$. No entanto, repovoamento desta área se dá em até 6 meses. A exposição à radiação UVA, atualmente utilizada de 5,4 $\mathrm{mJ} / \mathrm{cm}^{2}$, que corresponde a $3 \mathrm{~mW} / \mathrm{cm}^{2}$, está abaixo dos limiares de danos conhecidos do UVA para o endotélio corneano, cristalino e retina. Em relação ao dano fotoquímico causado pelos radicais livres, os limiares de danos para ceratócitos e células endoteliais são de 0,45 e $0,35 \mathrm{~mW} / \mathrm{cm}^{2}$, respectivamente. Em uma córnea de $400 \mu \mathrm{m}$ de espessura saturada com riboflavina, a irradiância no nível endotelial é de $0,18 \mathrm{~mW} / \mathrm{cm}^{2}$, o que é duas vezes menor do que o valor limite para ocorrer danos.

Assim, se a córnea, a ser tratada, tiver uma espessura mínima de $400 \mu \mathrm{m}$ (conforme recomendado), o endotélio corneano não sofrerá danos, nem estruturas mais profundas, como o cristalino e a retina [36]. Dessa forma, devido à semelhança dos resultados observados nos ensaios de citotoxicidade, do presente estudo, é necessário considerar a genipina como um reticulante promissor para o crosslinking do colágeno corneano.

\section{CONCLUSÕES}

A partir dos resultados encontrados, pode-se concluir que os ensaios de caracterização realizados em MEV e MO demostraram que a genipina $0,1 \%$, aplicada em córneas de porcos, promove crosslinking do colágeno, e que o efeito desse reticulante é semelhante ao produzido pelo protocolo padrão de CXL com riboflavina + UVA. Os ensaios de tração, dilatometria e grau de intumescimento não foram suficientes para avaliar qual dos métodos de reticulação apresenta maior efetividade. O potencial de citotoxicidade aos fibroblastos se mostrou semelhante entre os métodos de reticulação. Assim, novos estudos podem ratificar o benefício da genipina como reticulante do colágeno corneano no tratamento do ceratocone, tornando-a uma alternativa ao protocolo padrão de crosslinking no qual é necessário radiação ultravioleta.

\section{AGRADECIMENTOS}

Os autores agradecem ao Programa de Pós-Graduação em Ciência e Engenharia de Materiais (UAEMa), a Universidade Federal de Campina Grande (UFCG) e ao Laboratório de Avaliação e Desenvolvimento de Biomateriais do Nordeste (CERTBIO), pela oportunidade da realização deste trabalho. 


\section{BIBLIOGRAFIA}

[1] LAMY, R., NETTO, C.F., PECEGO, M.G., et al., "Reticulação do colágeno corneano com radiação ultravioleta e riboflavina para tratamento do ceratocone: resultados preliminares de um estudo brasileiro", Revista Brasileira Oftalmol, v. 67, n. 5, pp. 231-5, 2008.

[2] DAXER, A., MISOF, K., GRABNER, B., et al., "Collagen fibrils in the human corneal stroma: structure and aging". Investigative ophthalmology \& visual science, v. 39, n. 3, pp. 644-648, 1998.

[3] ANDRADE, C.T., COUTINHO, F.M.B., DIAS, M.L., et al., Dicionário de polímeros, Interciência, 2001.

[4] MANO, E.B., MENDES, L.C. Introdução a polímeros, São Paulo, Edgar Blücher, 1999.

[5] SPOERL, E., HUHLE, M., SEILER, T. "Induction of cross-links in corneal tissue", Experimental eye research, v. 66, n. 1, pp. 97-103, 1998.

[6] SEILER, T., SPOERL, E., HUHLE, M., et al., "Conservative therapy of keratoconus by enhancement of collagen cross-links", Investigative ophthalmology \& visual science, v. 37, n. 3, pp. 4671-4671, 1996.

[7] CONITEC, Relatório de Recomendação de Uso, 2016.

[8] BUTLER, M.F., NG, Y.F., PUDNEY, P.D.A. "Mechanism and kinetics of the crosslinking reaction between biopolymers containing primary amine groups and genipin", Journal of Polymer Science Part A: Polymer Chemistry, v. 41, n. 24, pp. 3941-3953, 2003.

[9] JIN, J., SONG, M., HOURSTON, D, "Novel chitosan-based films cross-linked by genipin with improved physical properties", Biomacromolecules, v. 5, n. 1, pp. 162-168, 2004.

[10] ONUKI, Y., BHARDWAJ, U., PAPADIMITRAKOPOULOS, F., et al., "A review of the biocompatibility of implantable devices: current challenges to overcome foreign body response", Journal of Diabetes Science and Technology, v. 2, n. 6, pp. 1003-1015, 2008.

[11] SUNG, H.W., CHANG, Y., CHIU, C.T., et al., "Crosslinking characteristics and mechanical properties of a bovine pericardium fixed with a naturally occurring crosslinking agent", Journal of Biomedical Materials Research Part A, v. 47, n. 2, pp. 116-126, 1999.

[12] AVILA, M.Y., GERENA, V.A., NAVIA, J.L. "Corneal crosslinking with genipin, comparison with UVriboflavin in ex-vivo model", Molecular vision, v. 18, pp. 1068, 2012.

[13] AVILA, M.Y., NAVIA, J.L. "Effect of genipin collagen crosslinking on porcine corneas", Journal of Cataract \& Refractive Surgery, v. 36, n. 4, pp. 659-664, 2010.

[14] Peschketrade, PXL PLATINUM 330 - MEDICAL DEVICE, http://www.peschketrade.com/products/peschke-cxl/. Acessado em maio de 2017.

[15] ARVO - Association for Research in Vision andOphthalmology, https://www.arvo.org/. Acessado em junho de 2017.

[16] CONCEA - Conselho Nacional de Controle de Experimentação Animal - Resolução Normativa, de 20 de setembro, 2013.

[17] ISO. Biological evaluation of medical devices - Part 5: Tests for in vitro cytotoxicity. ISO 10993-5. 2009.

[18] GARCIA, A., SPIM, J.A., SANTOS, C.A, Ensaios dos materiais, $2^{\mathrm{a}}$ ed, Livros Técnicos e científicos, 2000.

[19] HJORTDAL, J.O, "Regional elastic performance of the human cornea", Journal of biomechanics, v. 29, n. 7, pp. 931-942, 1996.

[20] KOTECHA, A. "What biomechanical properties of the cornea are relevant for the clinician?", Survey of ophthalmology, v. 52, n. 6, pp. S109-S114, 2007.

[21] HJORTDAL, J.O. "On the biomechanical properties of the cornea with particular reference to refractive surgery", Acta ophthalmologica Scandinavica, Supplement, n. 225, pp. 1-23, 1998.

[22] DANILOV, N., IGNATIEVA, N.Y., IOMDINA, E., et al., "Stabilization of scleral collagen by glycerol aldehyde cross-linking", Biochimica et Biophysica Acta (BBA)-General Subjects, v. 1780, n. 5, pp. 764-772, 2008.

[23] MANNHEIMER, W. A. Microscopia dos materiais: uma introdução. Rio de Janeiro: Editora E-papers, 2002.

[24] HERSH, P.S., STULTING, R.D., MULLER, D., et al., "United States multicenter clinical trial of corneal collagen crosslinking for keratoconus treatment", Ophthalmology, v. 124, n. 9, pp. 1259-1270, 2017. 
[25] GREENSTEIN, S.A., FRY, K.L., BHATT, J., et al., "Natural history of corneal haze after collagen crosslinking for keratoconus and corneal ectasia: Scheimpflug and biomicroscopic analysis", Journal of Cataract \& Refractive Surgery, v. 36, n. 12, pp. 2105-2114, 2010.

[26] KOLLER, T., MROCHEN, M., SEILER, T., "Complication and failure rates after corneal crosslinking", Journal of Cataract \& Refractive Surgery, v. 35, n. 8, pp. 1358-1362, 2009.

[27] SALOMÃO, M.Q., CHAURASIA, S.S., SINHA-ROY, A., et al., "Corneal wound healing after ultraviolet-A/riboflavin collagen cross-linking: a rabbit study", Journal of Refractive Surgery, v. 27, n. 6, pp. 401-407, 2011.

[28] BOTTÓS, K.M., DREYFUSS, J.L., REGATIERI, C.V., et al., "Immunofluorescence confocal microscopy of porcine corneas following collagen cross-linking treatment with riboflavin and ultraviolet A", Journal of Refractive Surgery, v. 24, n. 7, pp. S715-S719, 2008.

[29] SEILER, T., HAFEZI, F., "Corneal cross-linking-induced stromal demarcation line", Cornea, v. 25, n. 9, pp. 1057-1059, 2006.

[30] MAZZOTTA, C., BALESTRAZZI, A., TRAVERSI, C., et al., "Treatment of progressive keratoconus by riboflavin-UVA-induced cross-linking of corneal collagen: ultrastructural analysis by Heidelberg Retinal Tomograph II in vivo confocal microscopy in humans", Cornea, v. 26, n. 4, pp. 390-397, 2007.

[31] WOLLENSAK, J., BUDDECKE, E., "Biochemical studies on human corneal proteoglycans - a comparison of normal and keratoconic eyes", Graefe's archive for clinical and experimental ophthalmology, v. 228 , n. 6 , pp. 517-523, 1990.

[32] DINIZ, C.M., GADELHA, F.M., MICHELACCI, Y., et al., "Effects of Riboflavin and UV upon Glycosaminoglycan Synthesis in Human Keratoconic Corneas", Investigative ophthalmology \& visual science, v. 50, n. 13, pp. 5461-5461, 2009.

[33] WOLlENSAK, G., AURICH, H., PHAM, D.T., et al., "Hydration behavior of porcine cornea crosslinked with riboflavin and ultraviolet A", Journal of Cataract \& Refractive Surgery, v. 33, n. 3, pp. 516521, 2007.

[34] HAYES, S., KAMMA-LORGER, C.S., BOOTE, C., et al., "The effect of riboflavin/UVA collagen cross-linking therapy on the structure and hydrodynamic behaviour of the ungulate and rabbit corneal stroma", PLoS One, v. 8, n. 1, pp. e52860, 2013.

[35] WOLLENSAK, G., SPOERL, E., REBER, F., et al., "Keratocyte cytotoxicity of riboflavin/UVAtreatment in vitro", Eye, v. 18, n. 7, pp. 718-722, 2004.

[36] SPOERL, E., MROCHEN, M., SLINEY, D., et al., "Safety of UVA-riboflavin cross-linking of the cornea", Cornea, v. 26, n. 4, pp. 385-389, 2007.

\section{ORCID}

Camila Melo Gadelha Pereira Diniz https://orcid.org/0000-0003-2480-839X

Josué da Silva Buriti

Rossemberg Cardoso Barbosa

https://orcid.org/0000-0003-3207-2781

Marcus Vinicius Lia Fook

https://orcid.org/0000-0002-8551-5251

https://orcid.org/0000-0002-8566-920X 